November 1998

UCB-PTH-98/56

LBNL-42548

\title{
T-Duality and Ramond-Ramond Backgrounds in the Matrix Modelf
}

\author{
Daniel Bracef], Bogdan Morariuf and Bruno Zuminof \\ Department of Physics \\ University of California \\ and \\ Theoretical Physics Group \\ Lawrence Berkeley National Laboratory \\ University of California \\ Berkeley, California 94720
}

\begin{abstract}
We investigate T-duality of toroidally compactified Matrix model with arbitrary Ramond-Ramond backgrounds in the framework of noncommutative super Yang-Mills gauge theory.
\end{abstract}

*This work was supported in part by the Director, Office of Energy Research, Office of High Energy and Nuclear Physics, Division of High Energy Physics of the U.S. Department of Energy under Contract DE-AC03-76SF00098 and in part by the National Science Foundation under grant PHY-95-14797

†email address: brace@thwk2.lbl.gov

${ }_{\ddagger}^{\ddagger}$ email address: morariu@thsrv.lbl.gov

$\S$ email address: zumino@thsrv.lbl.gov 


\section{Introduction}

The original Matrix model conjecture [1], which required taking the large $n$ limit, was further refined in [2] by Susskind who conjectured that the discrete light-cone quantization (DLCQ) of M-theory is given by the finite $n$ Matrix model [3]. Upon compactification on a torus $T^{d}$ there are additional degrees of freedom, which correspond to winding states in the auxiliary Type IIA string theory [2, [4. The compactified Matrix model is described by a super Yang-Mills gauge theory on the dual torus. Alternatively, one could also ask directly if compactified Type IIA string theory in the $n$ D0-brane charge sector is equivalent to a $U(n)$ super Yang-Mills gauge theory. An equivalence between string theory and a finite rank gauge theory is only possible in the limit of decoupling string excitations which requires taking $\alpha^{\prime}$ to zero. To keep the winding excitations finite this implies we must also take the small volume limit. This is exactly the limit in which the Hamiltonian of the auxiliary Type IIA string theory equals the DLCQ Hamiltonian of M-theory [4].

For $d \geq 2$ besides the compactification metric there are additional moduli which, in terms of the auxiliary Type IIA string theory [4], correspond to the 2-form of the NS-NS (Neveu Schwarz-Neveu Schwarz) sector and the R-R (Ramond-Ramond) forms. In the seminal paper [5] Connes, Douglas and Schwarz conjectured that the 2-form of the NS-NS sector corresponds to the deformation parameter of a noncommutative super Yang-Mills (NCSYM) gauge theory. Further studies followed in [6, 0, 8, 9, 10, 11, 12].

In this paper we continue our investigation started in [9] of T-duality of the DLCQ of toroidally compactified M-theory and its realization in terms of NCSYM gauge theory. In [9] we gave an explicit description of the relationship proposed in [7, 河 between T-duality of string theory and the duality of the NCSYM gauge theory known in the mathematical literature as Morita equivalence [13]. Here we extend the previous results by allowing arbitrary R-R backgrounds. 
In general two NCSYM theories are dual to each other if there exists an element $\Lambda$ of the duality group $S O(d, d \mid \mathbf{Z})$ with the block decomposition

$$
\Lambda=\left(\begin{array}{ll}
\mathcal{A} & \mathcal{B} \\
\mathcal{C} & \mathcal{D}
\end{array}\right)
$$

such that their defining parameters are related as follows

$$
\begin{aligned}
\bar{\Theta} & =(\mathcal{A} \Theta+\mathcal{B})(\mathcal{C} \Theta+\mathcal{D})^{-1}, \\
\bar{G}^{i j} & =(\mathcal{C} \Theta+\mathcal{D})_{k}^{i}(\mathcal{C} \Theta+\mathcal{D})_{l}^{j} G^{k l}, \\
\bar{g}_{S Y M}^{2} & =\sqrt{|\operatorname{det}(\mathcal{C} \Theta+\mathcal{D})|} g_{S Y M}^{2}, \\
\bar{\eta} & =S(\Lambda) \eta \\
\bar{\chi} & =S(\Lambda) \chi .
\end{aligned}
$$

Here $S(\Lambda)$ denotes the Weyl spinor representation of $\Lambda$ and $\eta$ is an integral chiral spinor containing the Chern numbers of the bundle. For compactification on a three torus $\eta$ contains the rank of the group and the magnetic fluxes.

The first four relations can be found in [7, 8, 9]. Deriving (6) in the context of NCSYM gauge theory is the main thrust of our paper. The chiral spinor $\chi$ in (6) determines the parameters of the Chern-Simon type terms which we will add to the NCSYM action. In the auxiliary Type IIA string theory $\chi$ is closely related to the $\mathrm{R}$ - $\mathrm{R}$ moduli.

In Section 2 we review the transformation properties of the $\mathrm{R}-\mathrm{R}$ moduli under the duality group. The dimensionally reduced action of Type IIA supergravity is invariant under the T-duality group $S O(d, d)$. By deriving the nonlinear sigma model which describes the scalar fields of the supergravity, we can extract the transformation properties of the R-R backgrounds under the duality group. In particular we will show that appropriately defined

${ }^{a}$ The $S O(d, d \mid \mathbf{Z})$ subgroup of the T-duality group $O(d, d \mid \mathbf{Z})$ is the subgroup that does not exchange Type IIA and IIB string theories.

${ }^{b}$ The equations of motions are invariant under the full U-duality group $E_{d+1(d+1)}$. 
fields, which are combinations of the R-R forms and the NS-NS two-form, transform in a spinor representation of the duality group.

In Section 3, we identify the Chern-Simon parameters of the gauge theory with the R-R moduli. Then, we show that the duality transformations relating different NCSYM theories can be extended to include these terms. In the process we obtain the transformations properties of the parameters and show that they coincide with the transformations expected from string theory and derived in Section 2 using the dimensional reduction of 10-dimensional Type IIA supergravity.

Finally in the Appendix we present some results, used in the main text, regarding transformation properties under the T-duality group in the limit of small compactification volume and decoupling of string excitations.

After obtaining these results we received an e-print [10] containing a similar proposal for the additional terms in the noncommutative action.

\section{Duality of Seven Dimensional Supergravity}

Type IIA superstring theory compactified on a d-dimensional torus is invariant under the T-duality group $S O(d, d \mid \mathbf{Z})$. The low energy supergravity effective action describing this compactification is in fact invariant under the continuous group $S O(d, d)$. This action can be obtained directly from the 10-dimensional Type IIA supergravity by dimensional reduction. In this section we are interested in obtaining the transformation properties of the $\mathrm{R}-\mathrm{R}$ moduli under the discrete duality group. Since this is a subgroup of the corresponding continuous group which is a symmetry of the low energy 10-dimensional supergravity action, we can obtain these transformation properties by analyzing the symmetries of the the nonlinear sigma model which describes the dynamics of the scalars in the supergravity action.

The NS-NS scalars are described locally by an $O(d, d) / O(d) \times O(d)$ nonlinear sigma model. Taking into account the T-duality group, the NS-NS 
nonlinear sigma model is in fact defined on

$$
O(d, d \mid \mathbf{Z}) \backslash O(d, d) / O(d) \times O(d) .
$$

On the other hand simple counting arguments suggest that the R-R scalar fields transform in a chiral spinor representation of the duality group. This statement is almost correct except that the fields which transform in the spinor representation are some redefined fields involving not only the $\mathrm{R}-\mathrm{R}$ fields but also the NS-NS two form.

The 10-dimensional supergravity action written in terms of the string metric is given by

$$
\begin{gathered}
\mathcal{S}=\int d^{10} x \sqrt{g} e^{-2 \phi}\left(R+4(\nabla \phi)^{2}-\frac{1}{2 \cdot 3 !} H^{2}\right) \\
-\int d^{10} x \sqrt{g}\left(\frac{1}{2 \cdot 2 !} F^{2}+\frac{1}{2 \cdot 4 !} F^{2}\right) \\
-\frac{1}{4} \int F_{(4)} \wedge F_{(4)} \wedge B+\ldots,
\end{gathered}
$$

where we have not written the terms containing the fermionic fields. The first line contains only NS-NS fields while the second contains the kinetic terms of the R-R forms. The various field strengths are defined as follows

$$
\begin{aligned}
H & =d B, \\
F & =d A_{(1)}, \\
F_{(4)} & =d A_{(3)}, \\
F^{\prime} & =F_{(4)}+A_{(1)} \wedge d B,
\end{aligned}
$$

where the subscript indicates the rank of the form. Note that R-R fields couple to the NS-NS fields through the metric and through the $F^{\prime 2}$ term, which depends on the antisymmetric NS-NS two-form.

Next we perform the dimensional reduction along coordinates $x^{i}$ for $i=$ $1,2,3$. The massless scalars from the NS sector can be organized in the 
symmetric matrix [14]

$$
\mathcal{M}=\left(\begin{array}{cc}
G^{-1} & -G^{-1} B \\
B G^{-1} & G-B G^{-1} B
\end{array}\right) .
$$

Note that $\mathcal{M}$ is also an element of the group $S O(3,3)$. Using a result from the Appendix, we can obtain the Weyl spinor representation of $\mathcal{M}$

$$
S(\mathcal{M})=\left(\begin{array}{cc}
\operatorname{det} G^{-1 / 2} & \operatorname{det} G^{-1 / 2} b^{T} \\
\operatorname{det} G^{-1 / 2} b & \operatorname{det} G^{1 / 2} G^{-1}+\operatorname{det} G^{-1 / 2} b b^{T}
\end{array}\right),
$$

where $b=* B$, and the star denotes the operator which transforms an antisymmetric matrix into its dual column matrix. The star operator always dualizes only with respect to the compactified coordinates.

We obtain additional scalars from the dimensional reduction of $\mathrm{R}-\mathrm{R}$ forms. As mentioned above these fields do not have simple transformation properties under the T-duality group but we can define the following odd rank forms

$$
\begin{aligned}
& C_{(1)}=A_{(1)}, \\
& C_{(3)}=A_{(3)}-A_{(1)} \wedge B,
\end{aligned}
$$

and organize them in a column matrix which, as we will see shortly, transforms in a chiral spinor representation of the duality group

$$
\chi=\left(\begin{array}{c}
C_{123} \\
C_{1} \\
C_{2} \\
C_{3}
\end{array}\right) .
$$

The other fields can also be organized in representations of the duality group such that the action obtained by dimensional reduction from 10dimensional supergravity is explicitly invariant. The six vectors obtained from the dimensional reduction of NS-NS fields transform in the fundamental representation while the 7 -dimensional dilaton and the 7 -dimensional spacetime metric and 2-form are singlets. The four vectors obtained from the R-R 
forms transform in a chiral spinor representation and, after dualizing the 3 -form, the rest of the bosonic fields form a chiral spinor of 2-forms.

For our purpose, it will be enough to consider the nonlinear sigma model part of the action containing the kinetic terms of the scalar fields of the theory

$\mathcal{S}=\frac{1}{2} \int d^{7} x \sqrt{\widetilde{g}}\left(e^{-2 \Phi} \widetilde{g}^{\mu \nu} \operatorname{tr}\left(\partial_{\mu} \mathcal{M}^{-1} \partial_{\nu} \mathcal{M}\right)+\widetilde{g}^{\mu \nu} \partial_{\mu} \chi^{T} S(\mathcal{M}) \partial_{\nu} \chi\right)+\ldots$,

where $\widetilde{g}_{\mu \nu}$ and $\Phi$ are the 7-dimensional metric and dilaton, and we have not written the kinetic term for the dilaton. The nonlinear sigma model part of the action is written in a form that is explicitly invariant under $S O(3,3)$ and in fact the whole supergravity action could be written in invariant form. The duality transformations of the scalar fields are given by

$$
\begin{aligned}
\overline{\mathcal{M}} & =\Lambda^{-T} \mathcal{M} \Lambda^{-1} \\
\bar{\chi} & =S(\Lambda) \chi
\end{aligned}
$$

To prove the invariance of the action we used $S\left(\Lambda^{T}\right)=S(\Lambda)^{T}$.

The main purpose of this section was to obtain the relations (8) which show how the fields $\chi$ with simple transformations properties under the Tduality group are related to the R-R forms.

\section{T-duality of the Chern-Simon Type Terms}

In this section we show how to modify the NCSYM action so that it describes the DLCQ of M-theory in the presence of arbitrary moduli. In the auxiliary Type IIA string theory the additional moduli are constant R-R backgrounds corresponding to generalized Wilson lines. Then we show that the action which includes the new terms is also invariant under the duality group $S O(3,3 \mid \mathbf{Z})$ and that the parameters of the new terms transform exactly as expected from string theory. 
First we guess the form of these terms using our experience with the commutative case which corresponds to a vanishing NS-NS background 2form $B$. In this case the compactified Matrix model corresponding to $n$ D0-branes is described by a $U(n)$ supersymmetric Yang-Mills theory. This is obtained by performing a T-duality transformation along all the compact directions. However, for nonvanishing R-R moduli, the action contains an additional Chern-Simon type term [15]

$$
\mathcal{S}_{C S}=\frac{1}{4(2 \pi)^{3}} \int \operatorname{tr}\left(e^{2 \pi \mathcal{F}} \sum_{k \text { odd }} A^{(k)}\right),
$$

where $A^{(k)}$ are the the T-dual R-R fields. Note also that under T-duality in all directions the dual of $B$ also vanishes if $B$ was zero. This is why only $\mathcal{F}$ appears in the exponent while in general we would also subtract the dual of $B$.

Next we will consider the effect of a nonvanishing $B$ on this action. If $\gamma^{i j}$ represents a two cycle wrapped around directions $x^{i}$ and $x^{j}$, then the deformation parameters are defined by

$$
\Theta_{i j}=\frac{1}{(2 \pi)^{2}} \int_{\gamma^{i j}} B .
$$

In the super Yang-Mills part of the action the only change required by a nonvanishing $B$ was to make the coordinates noncommutative with deformation parameter $\Theta$. The metric and gauge coupling constant are the same as those obtained by T-duality from the Matrix model for a vanishing NS-NS 2-form. We emphasize that the metric of the NCSYM gauge theory is not the T-dual metric obtained by first taking the inverse of $E=G+\Theta$ and then extracting the symmetric part. The NCSYM metric $G^{i j}$ is just the inverse of the original metric. Thus we must distinguish between a T-duality in all directions and the noncommutative Fourier transformation relating the Matrix model and the NCSYM gauge theory.

Let us explain why the NCSYM metric is $\Theta$ independent. To compactify the Matrix model on a torus we first consider the Matrix model on the 
covering space and then impose a quotient condition. If the $B$ modulus is nonvanishing, once we go to the topologically trivial covering space, we can gauge it away. However this gauge transformation does not leave the wave functions of strings invariant and thus we must transform the translation operators implementing the quotient condition. The new translation operators do not commute and their noncomutativity is measured by $\Theta$.

Imposing the new quotient conditions on the Matrix action results directly in the NCSYM gauge theory. The only difference with the $B=0$ case is that we have to use noncommutative Fourier transformations instead of the standard Fourier transformations when we go from the Matrix model to the NCSYM gauge theory. This however does not result in a different metric and gauge coupling constant. The main point of this discussion was to show that we can trade a nonvanishing $B$ field for noncommutative coordinates on the dual super Yang-Mills gauge theory.

We will assume that the parameters of the Chern-Simon terms are also the same as for vanishing $\Theta$, except that the new terms are defined on a noncommutative torus. In particular for compactification on a three torus we have

$$
\mathcal{S}_{C S}=\frac{1}{4(2 \pi)^{3}} \int \operatorname{tr}\left(2 \pi \mathcal{F} \wedge A^{(2)}+\frac{1}{2} 2 \pi \mathcal{F} \wedge 2 \pi \mathcal{F} \wedge A^{(0)}\right) .
$$

Just as in the commutative case these terms are topological, supersymmetric and gauge invariant. In this action $\Theta$ only appears through the noncommutativity of the coordinates and $A^{(0)}$ and $A^{(2)}$ are the T-dual R-R formsf calculated as if the NS-NS 2-form vanishes

$$
A^{(0)}=* A_{(3)}, \quad A^{(2)}=-* A_{(1)} .
$$

The 1-form R-R field $A_{(1)}$ has a lower index and should not be confused with the Yang-Mills gauge field $A^{i}$. With this distinction in mind we can write the

${ }^{c}$ When we write the R-R forms in components we will drop the rank of the form as it is possible to identify the form from the position and number of indices. 
action (10) in the dual Matrix theory language using the R-R backgrounds on the original torus

$$
\mathcal{S}_{C S}=\int d t \operatorname{Tr}\left(\dot{X}^{i} A_{i}+\frac{i}{2 \pi} \dot{X}^{i} X^{j} X^{k} A_{i j k}\right),
$$

where $\mathrm{Tr}$ is the formal trace over infinite dimensional matrices divided by the infinite order of the quotient group [16]. It is convenient to write the action in component notation

$$
\mathcal{S}_{C S}=\frac{1}{2} \int d t \int \frac{d^{3} \sigma}{(2 \pi)^{3}} \operatorname{tr}\left(\varepsilon_{i j k}\left(2 \pi \mathcal{F}^{0 i} A^{j k}+(2 \pi)^{2} \mathcal{F}^{0 i} \mathcal{F}^{j k} A\right)\right),
$$

where the magnetic and electric field strengths in the temporal gauge are

$$
\mathcal{F}^{0 i}=i\left[\partial^{0}, D^{i}\right], \quad \mathcal{F}^{i j}=i\left[D^{i}, D^{j}\right] .
$$

We now show that the action (11) is invariant under the $S O(3,3 \mid \mathbf{Z})$ duality group of the auxiliary string theory. Consider a Chern-Simon type action defined on a $\eta$-bundle. Here $\eta$ is a $S O(3,3 \mid \mathbf{Z})$ spinor containing the rank of the group and the magnetic flux numbers

$$
\eta=\left(\begin{array}{c}
n \\
M^{23} \\
M^{31} \\
M^{12}
\end{array}\right) .
$$

We will perform the same sequence of field redefinitions used in [9], where it was shown explicitly for the case of vanishing R-R moduli, that the $U(n)$ NCSYM action is equivalent to a $U(q)$ NCSYM action on a trivial bundle, where $q$ is the greatest common divisor of $n$ and the magnetic fluxes $M$. Let $H=(\mathcal{C} \Theta+\mathcal{D})^{-1}$ be the matrix defined in [9], where $\mathcal{C}$ and $\mathcal{D}$ are the lower block components of the $S O(3,3 \mid \mathbf{Z})$ transformation relating the original NCSYM gauge theory to the theory on the trivial bundle with $U(q)$ gauge group. Then we make the following constant curvature connection and field redefinitions

$$
\widehat{\nabla}^{i} \stackrel{\text { def }}{=}\left(H^{-1}\right)_{j}^{i} \nabla^{j}, \widehat{A}^{i} \stackrel{\text { def }}{=}\left(H^{-1}\right)_{j}^{i} A^{j},
$$




$$
\begin{gathered}
\widehat{D}^{i} \stackrel{\text { def }}{=}\left(H^{-1}\right)_{j}^{i} D^{j}, \\
\widehat{\mathcal{F}}^{k l}=\left[\widehat{\nabla}^{k}, \widehat{A}^{l}\right]-\left[\widehat{\nabla}^{l}, \widehat{A}^{k}\right]-i\left[\widehat{A}^{k}, \widehat{A}^{l}\right] .
\end{gathered}
$$

The curvature can be split into a constant term and a fluctuating piece

$$
\begin{gathered}
\mathcal{F}^{i j}=\mathcal{F}_{(0)}^{i j}+H_{k}^{i} H_{l}^{j} \widehat{\mathcal{F}}^{k l}, \\
\mathcal{F}^{0 k}=H_{l}^{k} \widehat{\mathcal{F}}^{0 l} .
\end{gathered}
$$

Using the matrices $Q$ and $R$ defined in [9] we perform a change of integration variables $\widehat{\sigma}=\sigma Q R$, which introduces a Jacobian factor

$$
\int d^{3} \sigma \operatorname{tr} \Psi(\sigma)=\int d^{3} \widehat{\sigma} \operatorname{det}\left(Q^{-1}\right) \operatorname{tr} \Psi\left(\widehat{\sigma}(Q R)^{-1}\right) .
$$

Making the substitutions (12), (13) and collecting similar terms we find

$$
\mathcal{S}_{C S}=\frac{1}{2(2 \pi)^{2}} \int d t \int d^{3} \widehat{\sigma} \frac{q}{n} \operatorname{tr}\left(\varepsilon_{i j k}\left(\widehat{\mathcal{F}}^{0 i} A^{\prime j k}+2 \pi \widehat{\mathcal{F}}^{0 i} \widehat{\mathcal{F}}^{j k} A^{\prime}\right)\right),
$$

where

$$
\begin{gathered}
q A^{\prime}=\left(n \operatorname{det} Q^{-1} \operatorname{det} H\right) A, \\
\varepsilon_{i j k} q A^{\prime i j}=H_{k}^{l} \varepsilon_{i j l} n \operatorname{det} Q^{-1}\left(A^{i j}+2 \pi \mathcal{F}_{(0)}^{i j} A\right) .
\end{gathered}
$$

One can now rewrite the action in terms of new operators $\sigma_{i}^{\prime}$, $\partial^{\prime i}$, and $U^{\prime}$, and a $q$ dimensional trace. See [9] for a more detailed discussion of this substitution.

$$
\mathcal{S}_{C S}=\frac{1}{4(2 \pi)^{3}} \int \operatorname{tr}_{q}\left(2 \pi \mathcal{F}^{\prime} \wedge A^{\prime(2)}+\frac{1}{2} 2 \pi \mathcal{F}^{\prime} \wedge 2 \pi \mathcal{F}^{\prime} \wedge A^{\prime(0)}\right)
$$

More generally the action is invariant under duality transformations if the Chern-Simon parameters are related as follows

$$
\begin{aligned}
\bar{A}_{(0)} & =|\operatorname{det}(\mathcal{C} \Theta+\mathcal{D})|^{-1 / 2} A_{(0)}, \\
*\left(\bar{A}^{(2)}+2 \pi \overline{\mathcal{F}}_{(0)} \bar{A}^{(0)}\right) \bar{n} \operatorname{det} \bar{Q}^{-1} & =(\mathcal{C} \Theta+\mathcal{D})^{-T} *\left(A^{(2)}+2 \pi \mathcal{F}_{(0)} A^{(0)}\right) n \operatorname{det} Q^{-1},
\end{aligned}
$$


where $\mathcal{C}$ and $\mathcal{D}$ are the lower block components of the $S O(3,3 \mid \mathbf{Z})$ matrix relating the two theories, and the star operator is the duality operator acting only with respect to the compact coordinates.

Next we write the Chern-Simon parameters in term of the fields $C$ discussed in Section 2

$$
\begin{gathered}
A^{(0)}=*\left(C_{(3)}+C_{(1)} \wedge \Theta\right), \\
A^{(2)}=-* C_{(1)} .
\end{gathered}
$$

To obtain a compact form first define the column matrices $u$ and $v$ with components

$$
\begin{aligned}
u_{i} & =n C_{i}-\frac{1}{2} M^{j k} C_{i j k}, \\
v^{i} & =M^{i j} C_{j} .
\end{aligned}
$$

If $\chi$ transforms as a spinor, $u$ and $v$ are the block components of a $S O(3,3 \mid \mathbf{Z})$ vector as shown in the Appendix (27). Then using the identities (28) listed in the Appendix, the transformation (14) can be written as

$$
\begin{gathered}
\bar{C}_{(3)}+\bar{C}_{(1)} \wedge \bar{\Theta}=|\operatorname{det}(\mathcal{C} \Theta+\mathcal{D})|^{-1 / 2}\left(C_{(3)}+C_{(1)} \wedge \Theta\right), \\
(\bar{u}-\bar{\Theta} \bar{v})=(\mathcal{C} \Theta+\mathcal{D})^{-T}(u-\Theta v) .
\end{gathered}
$$

Comparing (17) and (18) with (25) and (23) in the Appendix we see that the $\mathrm{R}-\mathrm{R}$ fields must transform in a spinor representation of $S O(3,3 \mid \mathbf{Z})$

$$
\bar{\chi}=S(\Lambda) \chi
$$

Thus the duality transformations of all the parameters of the NCSYM, including those of the Chern-Simon type terms, coincide with the transformation of moduli of the Type IIA strings compactified on a torus in the limit of vanishing $\alpha^{\prime}$ and $G_{i j}$.

Using the transformation properties of $g_{S Y M}^{-2}$ and $A^{(0)}$ it follows that the complex coupling

$$
\tau=A^{(0)}+\frac{4 \pi i}{g_{S Y M}^{2}}
$$


also transforms simply under the T-duality group with the same $\Theta$ dependent factor appearing in (14).

Finally note that the BPS spectrum corresponding to the electric fluxes obtained in [10, 11, 12] is modified in the presence of nonvanishing R-R moduli

$$
\begin{gathered}
\mathcal{E}^{U(n)}=\frac{g_{S}}{2}\left|n-\frac{1}{2} \operatorname{tr}(M \Theta)\right|^{-1} \\
\times\left(\left(n_{i}-u_{i}\right)-\Theta_{i k}\left(m^{k}-v^{k}\right)\right) G^{i j}\left(\left(n_{j}-u_{j}\right)-\Theta_{j l}\left(m^{l}-v^{l}\right)\right),
\end{gathered}
$$

where we used the notation of [11].

\section{Acknowledgments}

This work was supported in part by the Director, Office of Energy Research, Office of High Energy and Nuclear Physics, Division of High Energy Physics of the U.S. Department of Energy under Contract DE-AC03-76SF00098 and in part by the National Science Foundation under grant PHY-95-14797.

\section{Appendix}

In this Appendix we present some mathematical results regarding the spinor representation of the T-duality group and duality invariant quantities in the small volume limit. The group $S O(d, d)$ is the group of $2 d$-dimensional matrices $\Lambda$ satisfying $\Lambda J \Lambda^{T}=J$ where $J$ is a matrix with the block form

$$
J=\left(\begin{array}{ll}
0 & 1 \\
1 & 0
\end{array}\right)
$$

It will be useful to know how to calculate the Weyl spinor representation matrix of an $S O(3,3)$ group element $\Lambda$ with the block form

$$
\Lambda=\left(\begin{array}{ll}
\mathcal{A} & \mathcal{B} \\
\mathcal{C} & \mathcal{D}
\end{array}\right)
$$


First note that if $\mathcal{A}$ is invertible $\Lambda$ has a block Gauss decomposition

$$
\Lambda=\left(\begin{array}{cc}
1 & 0 \\
\mathcal{C A}^{-1} & 1
\end{array}\right)\left(\begin{array}{cc}
\mathcal{A} & 0 \\
0 & \mathcal{A}^{-T}
\end{array}\right)\left(\begin{array}{cc}
1 & \mathcal{A}^{-1} \mathcal{B} \\
0 & 1
\end{array}\right)
$$

where one can show using the group relations that $\mathcal{C A}^{-1}$ and $\mathcal{A}^{-1} \mathcal{B}$ are antisymmetric. This decomposition is in fact true for generic $S O(d, d)$ matrices. For $d=3$ we can give the explicit spinor representation matrices for each factor in (7) thus obtaining the spinor representation of a generic $S O(3,3)$ matrix $\Lambda$

$$
S=\left(\begin{array}{cc}
1 & 0 \\
*\left(\mathcal{C A}^{-1}\right) & 1
\end{array}\right)\left(\begin{array}{cc}
\operatorname{det} \mathcal{A}^{1 / 2} & 0 \\
0 & \operatorname{det} \mathcal{A}^{-1 / 2} \mathcal{A}
\end{array}\right)\left(\begin{array}{cc}
1 & *\left(\mathcal{A}^{-1} \mathcal{B}\right)^{T} \\
0 & 1
\end{array}\right) .
$$

The star denotes the duality operator. When acting on antisymmetric 3dimensional square matrices it gives the dual column matrix.

We can form invariants using two column matrices transforming in the vector representation of $S O(d, d \mid \mathbf{Z})$ and the symmetric $S O(d, d \mid \mathbf{Z})$ matrix $\mathcal{M}$

$$
\left(r^{T}, s^{T}\right) \mathcal{M}\left(\begin{array}{l}
u \\
v
\end{array}\right) .
$$

In the limit when $G$ goes to zero, using the block Gauss decomposition of $\mathcal{M}$

$$
\mathcal{M}=\left(\begin{array}{ll}
1 & 0 \\
B & 1
\end{array}\right)\left(\begin{array}{cc}
G^{-1} & 0 \\
0 & G
\end{array}\right)\left(\begin{array}{cc}
1 & -B \\
0 & 1
\end{array}\right),
$$

and after identifying $B$ with $\Theta$ we obtain the following invariant

$$
(r-\Theta s)^{T} G^{-1}(u-\Theta v) .
$$

Using the transformation of $G$ under the duality group (3) we can write the transformation of $u-\Theta v$

$$
(\bar{u}-\bar{\Theta} \bar{v})=(\mathcal{C} \Theta+\mathcal{D})^{-T}(u-\Theta v) .
$$

\footnotetext{
${ }^{d}$ To obtain a finite result, one should insert appropriate factors of $\alpha^{\prime}$ in (22) and also take $\alpha^{\prime}$ to zero as discussed in the Introduction.
} 
The spinor representation matrix of $\mathcal{M}$ can be calculated using (21)

$$
S(\mathcal{M})=\left(\begin{array}{ll}
1 & 0 \\
b & 1
\end{array}\right)\left(\begin{array}{cc}
\operatorname{det} G^{-1 / 2} & 0 \\
0 & \operatorname{det} G^{1 / 2} G^{-1}
\end{array}\right)\left(\begin{array}{cc}
1 & b^{T} \\
0 & 1
\end{array}\right) .
$$

Then we can also form the invariants $\eta^{T} S(\mathcal{M}) \chi$ using two chiral spinors

$$
\chi=\left(\begin{array}{l}
\chi_{0} \\
\chi_{1} \\
\chi_{2} \\
\chi_{3}
\end{array}\right), \quad \eta=\left(\begin{array}{l}
\eta_{0} \\
\eta_{1} \\
\eta_{2} \\
\eta_{3}
\end{array}\right) .
$$

In the limit of vanishing $G$, the invariant becomes

$$
\left(\eta_{0}+\frac{1}{2} \varepsilon^{i j k} \Theta_{i j} \eta_{k}\right) \sqrt{\operatorname{det} G^{-1}}\left(\chi_{0}+\frac{1}{2} \varepsilon^{i j k} \Theta_{i j} \chi_{k}\right) .
$$

From (24) we obtain the following transformation law

$$
\bar{\chi}_{0}+\frac{1}{2} \varepsilon^{i j k} \bar{\Theta}_{i j} \bar{\chi}_{k}=|\operatorname{det}(\mathcal{C} \Theta+\mathcal{D})|^{-1 / 2}\left(\chi_{0}+\frac{1}{2} \varepsilon^{i j k} \Theta_{i j} \chi_{k}\right) .
$$

One can also check the relations (23) and (25) directly using the trasformations (国) of $\Theta$.

Given two chiral spinors $\chi$ and $\eta$ we first write them as Dirac spinors

$$
\chi_{D}=\left(\begin{array}{c}
\chi \\
0
\end{array}\right), \quad \eta_{D}=\left(\begin{array}{c}
\eta \\
0
\end{array}\right) .
$$

Then using the same definition for $a^{i}$ and $a_{i}^{\dagger}$ as in the Appendix of [9] we can form the $S O(3,3 \mid \mathbf{Z})$ vector

$$
\left(\begin{array}{c}
u_{i} \\
v^{i}
\end{array}\right)=\bar{\eta}_{D}\left(\begin{array}{c}
a_{i}^{\dagger} \\
a^{i}
\end{array}\right) \chi_{D} .
$$

where $\bar{\eta}_{D}=\eta^{\dagger} T$. Here $T$ is a matrix acting on Dirac spinors and plays the same role as $\gamma_{0}$ when we form barred spinors in Minkowski space. It is given by

$$
T=\left(a_{1}^{\dagger}+a^{1}\right)\left(a_{2}^{\dagger}+a^{2}\right)\left(a_{3}^{\dagger}+a^{3}\right) .
$$


Writing out all the spinor components in (26) we have

$$
\left(\begin{array}{c}
u_{i} \\
v^{i}
\end{array}\right)=\left(\begin{array}{c}
\eta_{i} \chi_{0}-\eta_{0} \chi_{i} \\
\varepsilon^{i j k} \eta_{j} \chi_{k}
\end{array}\right) .
$$

Then $u-\Theta v$ transforms as in (23) under the duality group. Such an expression, involving two chiral spinors and $\Theta$, is used in the main text.

Finally we list some useful identities presented in [9]

$$
\begin{aligned}
\operatorname{det} H & =(q \operatorname{det}(Q) / n)^{2}, \\
M & =2 \pi n Q^{-1} \mathcal{F}_{(0)} Q^{-T}, \\
M^{i j} \varepsilon_{i j l} & =M^{i j} \varepsilon_{i j k} Q_{l}^{k} .
\end{aligned}
$$

\section{References}

[1] T. Banks, W. Fishler, S.H. Shenker, L Susskind, M Theory As A Matrix Model: A Conjecture, Phys. Rev. D55 (1997) 5112-5128; hep-th/9610043

[2] L. Susskind, Another Conjecture about M(atrix) Theory, hep-th/9704080

[3] M. Claudson, M. Halpern, Supersymmetric Ground State Wave Functions, Nucl. Phys. B250 (1985) 689

R. Flume, On Quantum Mechanics with Extended Supersymmetry and Nonabelian Gauge Constraints, Ann. Phys. 164 (1985) 189

M. Baake, P. Reinicke, V. Rittenberg, Fierz Identities for Real Clifford Algebras and the Number of Supercharges, J. Math. Phys. 26 (1985) 1070

[4] A. Sen, D0 Branes on $T^{n}$ and Matrix Theory, Adv. Theor. Math. Phys. 2 (1998) 51-59; hep-th/9709220

N. Seiberg, Why is the Matrix Model Correct?, Phys. Rev. Lett. 79 (1997) 3577-3580; hep-th/9710009 
[5] A. Connes, M. R. Douglas, A. Schwarz, Noncommutative Geometry and Matrix Theory: Compactification on Tori, JHEP02(1998)003; hepth/9711162

[6] M. R. Douglas, C. Hull, D-branes and the Noncommutative Torus, JHEP02 (1998) 008; hep-th/9711165

P.-M. Ho, Y.-Y. Wu, Y.-S. Wu, Towards a Noncommutative Geometric Approach to Matrix Compactification, hep-th/9712201

P.-M. Ho, Y.-S. Wu, Noncommutative Gauge Theories in Matrix Theory, hep-th/9801147

R. Casalbuoni, Algebraic Treatment of Compactification on Noncommutative Tori, hep-th/9801170

Y.-K. E. Cheung, M. Krogh, Noncommutative Geometry from 0-branes in a Background B-field, hep-th/9803031

T. Kawano, K. Okuyama, Matrix Theory on Noncommutative Torus, hep-th/9803044

F. Ardalan, H. Arfaei, M. M. Sheikh-Jabbari, Mixed Branes and M(atrix) Theory on Noncommutative Torus, hep-th/9803067

P.-M. Ho, Twisted Bundle on Quantum Torus and BPS States in Matrix Theory, hep-th/9803166

D. Bigatti, Non commutative geometry and super Yang-Mills theory, hep-th/9804120

G. Landi, F. Lizzi, R. J. Szabo, String Geometry and Noncommutative Torus, hep-th/9806099

B. Morariu, B. Zumino, Super Yang-Mills on the Noncommutative Torus, hep-th/9807198

F. Ardalan, H. Arfaei, M. M. Sheikh-Jabbari, Noncommutative Geometry from Strings and Branes, hep-th/9810072

[7] M. Rieffel, A. Schwarz, Morita equivalence of multidimensional noncommutative tori, math.QA/98033057 
[8] A. Schwarz, Morita equivalence and duality, hep-th/9805034

[9] D. Brace, B. Morariu, B. Zumino, Dualities of the Matrix Model from T-Duality of the Type II String, hep-th/9810099

[10] C. Hofman, E. Verline Gauge Bundles and Born-Infeld on the Noncommutative Torus, hep-th/9810219

[11] D. Brace, B. Morariu, A note on the BPS Spectrum of the Matrix Model, hep-th/9810185

[12] A. Konechny, A. Schwarz BPS states on noncommutative tori and duality, hep-th/9811159

[13] M. A. Rieffel, Induced Representations of $C^{*}$-algebras, Advances Math. 13 (1974) 176-257

M. A. Rieffel, $\mathbf{C}^{*}$-Algebras Associeted with Irrational Rotations, Pacific J. Math. 93 (1981) 415-429

M. A. Rieffel, The Cancellation Theorem for Protective Modules over Irrational Rotations $\mathbf{C}^{*}$-Algebras, Proc. London Math. Soc. (3), 47 (1983) 285-302

M. A. Rieffel, Morita Equivalence for Operator Algebras, Operator Algebras and Applications, (R. V. Kadison, ed.) Proc. Symp. Pure Math. 38, Amer. Math. Soc., Providence, 1982

M. A. Rieffel, Projective Modules over Higher-Dimensional Noncommutative Tori, Can. J. Math., XL No. 2 (1988) 257-338

[14] A. Sen, Strong-Weak Coupling Duality in Four Dimensional String Theory, Int. J. Mod. Phys. A9 (1994) 3707-3750; hep-th/9402002

[15] M. Li, Boundary States of D-Branes and Dy-Strings Nucl. Phys. B460 (1996) 351; hep-th/9510161

[16] W. Taylor IV, D-brane Field Theory on Compact Spaces, Phys. Lett. B394 (1997) 283; hep-th/9611042 